\title{
Maternal hemoglobin associates with preterm delivery and small for gestational age in two Finnish birth cohorts
}

\section{Ronkainen, Justiina}

2019-07

Ronkainen , J , Lowry , E , Heiskala , A , Uusitalo , L , Koivunen , P , Kajantie , E , Vääräsmäki , M , Järvelin , M-R \& Sebert , S 2019 , ' Maternal hemoglobin associates with preterm delivery and small for gestational age in two Finnish birth cohorts ' , European Journal of Obstetrics, and Gynecology ,and Reproductive Biology , vol. 238 , pp. 44-48 . https://doi.org/10.1016/j.ejo

http://hdl.handle.net/10138/318819

https://doi.org/10.1016/j.ejogrb.2019.04.045

publishedVersion

Downloaded from Helda, University of Helsinki institutional repository.

This is an electronic reprint of the original article.

This reprint may differ from the original in pagination and typographic detail.

Please cite the original version. 


\title{
Maternal hemoglobin associates with preterm delivery and small for gestational age in two Finnish birth cohorts
}

\author{
Justiina Ronkainen ${ }^{\mathrm{a}, \mathrm{b}, *}$, Estelle Lowry ${ }^{\mathrm{a}, \mathrm{b}}$, Anni Heiskala ${ }^{\mathrm{a}, \mathrm{b}}$, Iida Uusitalo ${ }^{\mathrm{a}, \mathrm{b}}$, \\ Peppi Koivunen ${ }^{b, c, d}$, Eero Kajantie e,f,g,h , Marja Vääräsmäki ${ }^{e, f}$, Marjo-Riitta Järvelinn $^{\mathrm{a}, \mathrm{b}, \mathrm{i}, \mathrm{j}}$, \\ Sylvain Sebert ${ }^{\mathrm{a}, \mathrm{b}, \mathrm{k}}$
}

a Center for Life Course Health Research, University of Oulu, Oulu, Finland

${ }^{\mathrm{b}}$ Biocenter Oulu, University of Oulu, Oulu, Finland

${ }^{\mathrm{c}}$ Faculty of Biochemistry and Molecular Medicine, University of Oulu, Oulu, Finland

d Oulu Center for Cell-Matrix Research, University of Oulu, Oulu, Finland

e PEDEGO Research Unit, MRC Oulu, Oulu University Hospital and University of Oulu, Oulu, Finland

${ }^{\mathrm{f}}$ Public Health Promotion Unit, Department of Public Health Solutions, National Institute for Health and Welfare, Helsinki, Finland

${ }^{g}$ Hospital for Children and Adolescents, Helsinki University Central Hospital and University of Helsinki, Helsinki, Finland

${ }^{\mathrm{h}}$ Department of Clinical and Molecular Medicine, Norwegian University of Science and Technology, Trondheim, Norway

${ }^{i}$ Department of Epidemiology and Biostatistics, School of Public Health, Imperial College London, London, United Kingdom

${ }^{\mathrm{j}}$ MRC-PHE Centre for Environment and Health, School of Public Health, Imperial College London, London, United Kingdom

${ }^{\mathrm{k}}$ Department for Genomics of Common Diseases, School of Medicine, Imperial College London, London, United Kingdom

\section{A R T I C L E I N F O}

\section{Article history:}

Received 20 February 2019

Received in revised form 27 March 2019

Accepted 29 April 2019

\section{Keywords:}

Intrauterine growth restriction

Large for gestational age

Maternal hemoglobin

Preterm delivery

Small for gestational age

\begin{abstract}
A B S T R A C T
Objective: To test whether maternal hemoglobin during pregnancy associates with offspring perinatal outcomes in a developed country. Changes in maternal hemoglobin concentration during pregnancy are partly physiological phenomena reflecting alterations of maternal blood volume. Especially hemoglobin measures outside the physiological range may influence maternal health and fetal growth with longlasting consequences.

Study design: We studied an unselected sample drawn from two regional birth cohorts born 20 years apart: The Northern Finland Birth Cohorts 1966 and 1986. These are two mother-and-child populationbased birth cohorts together comprising 21,710 mothers and their children. After exclusions, the sample size of the current study was 20,554. Concentrations of maternal hemoglobin at first and last antenatal visits were categorized as low (lowest 10\%), medium (reference) or high (highest 10\%). Multinomial logistic regression analyses for categories of maternal hemoglobin and perinatal outcomes such as preterm delivery and full-term small and large for gestational age were conducted with adjustments for maternal cofactors.

Results: Low maternal hemoglobin at early pregnancy associated with decreased risk of full-term small for gestational age (adjusted $\mathrm{OR} 0.73,95 \% \mathrm{CI}[0.58,0.93], \mathrm{p}=0.010$ ). At late pregnancy, low maternal hemoglobin associated with increased risk of preterm delivery (adjusted OR 1.60, 95\% CI [1.26, 2.02], $\mathrm{p}<0.0005$ ) whereas high maternal hemoglobin associated with increased risk of full-term small for gestational age (adjusted OR 1.29, 95\% CI [1.07, 1.56], $\mathrm{p}=0.009$ ). Maternal hemoglobin did not show constant association with risk of large for gestational age.

Conclusion: The results from this study support evidence that both low and high maternal hemoglobin associate with adverse perinatal outcomes. Low maternal hemoglobin associated with preterm delivery and high with full-term small for gestational age. Association was mainly present when maternal hemoglobin was measured during the third trimester. These results indicate that it is important to monitor both extremes of maternal hemoglobin throughout the pregnancy.
\end{abstract}

(c) 2019 Elsevier B.V. All rights reserved.

\footnotetext{
* Corresponding author at: PO BOX 5000, FI-90014, Oulu, Finland.

E-mail address: justiina.ronkainen@oulu.fi (J. Ronkainen).
}

\section{Introduction}

Both low and high maternal hemoglobin $(\mathrm{mHb})$ concentration has been consistently associated with adverse perinatal outcomes 
such as preterm birth and fetal growth restriction [1-7] and in our own data with later developmental indices [8]. Low hemoglobin and anemia (generally defined as $\mathrm{mHb}<110 \mathrm{~g} / \mathrm{l}$ ) are global problems affecting both the mother and the developing child. In 2011, the global prevalence of anemia among pregnant women was $38 \%$, which translated to 32 million mothers [9]. Regionally, the prevalence of maternal anemia vary substantially from $22 \%$ in European and North American countries to $56 \%$ in Central and West Africa [10].

Several environmental and behavioral maternal factors affect $\mathrm{mHb}$ and the developing child. Smoking during pregnancy has been associated with placental defects, prematurity and growth restriction (reviewed in [11]). Furthermore, smoking associates with increased mHb levels, which may contribute to increased risk of adverse perinatal outcomes [7,12]. Low maternal BMI is associated with increased risk of spontaneous preterm birth whereas some studies report obesity to have a protective effect [13]. However, obese mothers are in risk for hypertensive disorders, which associate with increased risk of medically indicated preterm birth [13]. Mother's low education was associated with prematurity and small for gestational age (SGA) in recent meta-analysis comprising of 12 European countries, although there were differences between countries [14]. Nulliparous mothers are at increased risk of preterm delivery and SGA specifically if the mother is under 18 years old and maternal age 35 or more is associated with increased risk of SGA but not prematurity [15].

In the current study, we study the association between $\mathrm{mHb}$ and adverse perinatal outcomes within two study populations located in Finland. These populations are highly homogenous due to standardized antenatal care and pregnancy counselling, which contributes to fewer environmental confounders than in more diverse settings. Association between $\mathrm{mHb}$ at different time points of pregnancy and such outcomes as prematurity and small and large for gestational age (SGA and LGA) are studied.

\section{Material and methods}

\section{Inclusion and exclusion criteria}

The study population is the mothers and children of Northern Finland Birth Cohorts (NFBC) 1966 and 1986 which are populationbased birth cohorts comprising $98 \%(n=21,710)$ of all deliveries occurred in 1966 and 1985-86 in Oulu and Lapland provinces of Finland. Multiple pregnancies, individuals without both early and late $\mathrm{mHb}$ measurements and individuals with $\mathrm{mHb}$ over $5 \mathrm{SD}$ from mean $(n=1,156)$ were excluded from the analyses resulting in final

Table 1

Characteristics of mothers and singleton newborns in the pooled sample and stratified by the cohort.

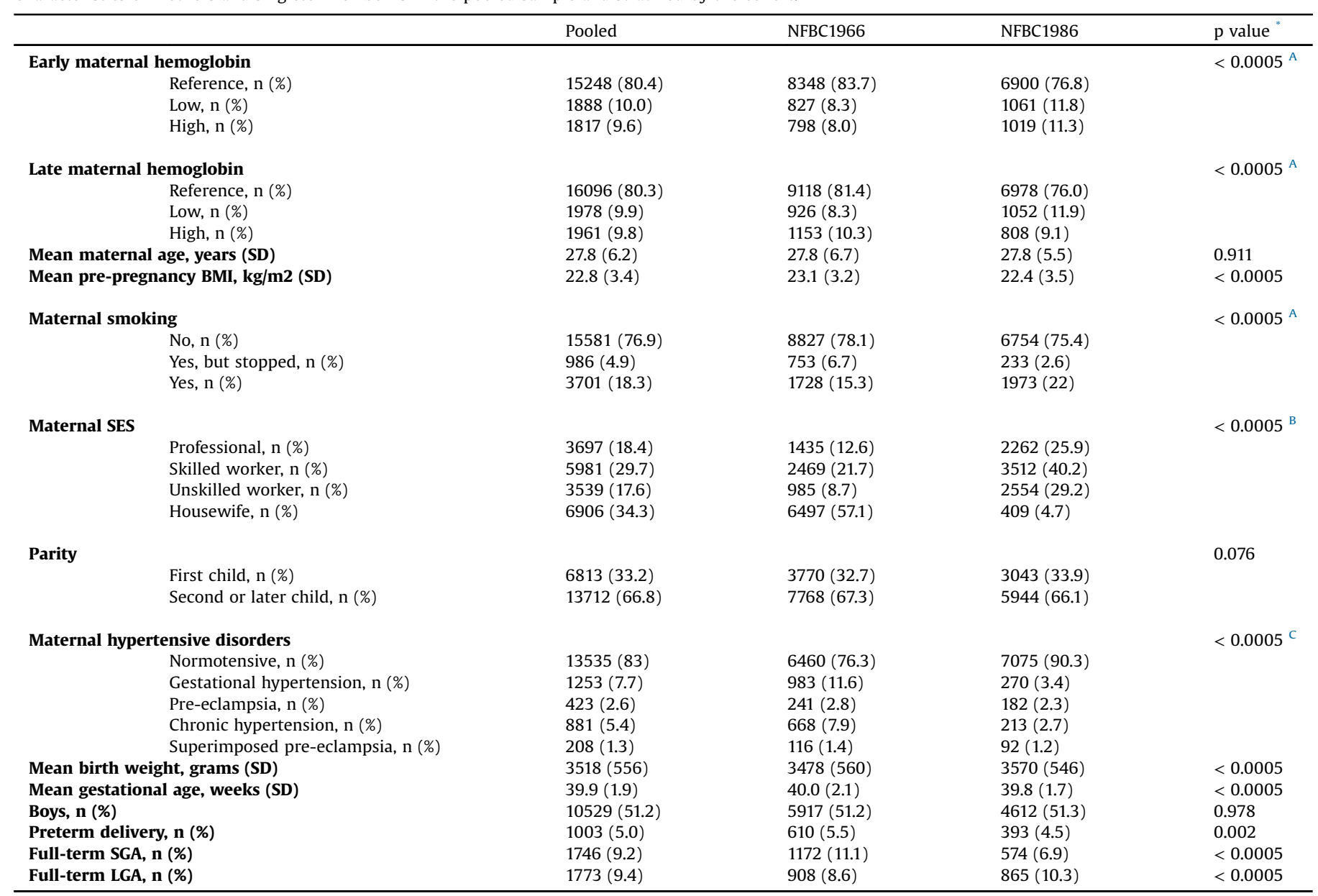

* Comparison between NFBC1966 and NFBC1986.

A $\mathrm{p}<0.05$ between all $\mathrm{mHb}$ categories.

B $\mathrm{p}<0.05$ between all SES categories except professional vs. skilled worker.

C $\mathrm{p}<0.05$ between all hypertensive disorder categories except normotensive vs. superimposed pre-eclampsia, gestational hypertension vs. chronic hypertension and preeclampsia vs. superimposed pre-eclampsia. 
sample size of 20,554 . Concentration of $\mathrm{mHb}$ at early pregnancy was available from 18,953 mothers and at late pregnancy from 20,035 mothers. Informed consent was obtained from study subjects for the use of their data in the study. Approval for the studies was granted by the ethics committee of the Northern Ostrobothnia Hospital District in Oulu, Finland in accordance with the declaration of Helsinki.

\section{Predictor variables}

Mean gestational week for early mHb measurement was 13.3 (SD 4.8) and for late mHb 38.2 (SD 3.4). In NFBC1966, mHb was measured with Tallquist chart, hemometer or photometer and in NFBC1986, with standard photometer. In NFBC1966, mHb was first standardized for measurement method and then $\mathrm{mHb}$ measures were transformed to $\mathrm{z}$ values $[\mathrm{z}(\mathrm{mHb})=(\mathrm{mHb}-\operatorname{mean}(\mathrm{mHb})) / \mathrm{SD}$ $(\mathrm{mHb})$ ] separately for each cohort and pregnancy time point. $\mathrm{Z}$ value of $\mathrm{mHb}$ below 10th percentile was defined as low and above 90th percentile as high $\mathrm{mHb}$ while the middle $80 \%$ was used as reference.

\section{Perinatal outcomes}

Children born before 37 weeks of gestation were defined as preterm. SGA and LGA were analyzed from full-term children (gestational age 37 weeks or more) only. SGA was defined as below 10th percentile and LGA as above 90th percentile of the Finnish national reference according to [16].

\section{Covariates}

Following factors were used as covariates in the statistical models: maternal smoking, maternal hypertensive disorders, maternal pre-pregnancy body mass index (BMI), maternal socioeconomic status (SES), parity and maternal age. Maternal smoking was categorized as not smoked during pregnancy (reference, $n=15,581$ ), smoked, but not after second month of pregnancy $(n=986)$ and smoked after second month of pregnancy $(n=3,701)$. Maternal hypertensive disorders were defined as described in [17]: Normotensive (reference, $n=13,535)$, gestational hypertension $(n=1,253)$, pre-eclampsia $(\mathrm{n}=423)$, chronic hypertension $(\mathrm{n}=881)$ and superimposed preeclampsia $(n=208)$. Maternal SES was categorized as professionals (reference, $n=3,697$ ), skilled workers ( $n=5,981$ ), unskilled workers $(n=3,539)$ and housewives, farmers and farmer's wives $(n=6,906)$. Parity was dichotomized as nulliparous (reference, $n=6,813$ ) and multiparous $(n=13,712)$. Maternal pre-pregnancy BMI (weight in $\mathrm{kg} /(\text { height in } \mathrm{m})^{2}$ ) and age were used as continuous variables. Cohort was included in the models, NFBC1966 as a reference $(n=11,554)$ and NFBC1986 $(n=9,000)$. Sex of the child was not included in the models because it did not associate with prematurity in the current population and SGA and LGA were determined from sex specific growth curves [16].

\section{Statistics}

Data from two cohorts were pooled. Differences in maternal and fetal characteristics between the cohorts were analyzed with independent samples $t$-test for continuous variables and chi squared test of independence with subsequent post hoc analyses for categorical variables. Association between $\mathrm{mHb}$ at different time points of pregnancy and perinatal outcomes was analyzed using multinomial logistic regression (MLR). Odds ratios (OR) and 95\% confidence intervals (95\% CI) were recorded and p-values according to Wald's test that were below 0.0125 (0.05/4 tests) were considered statistically significant. Association was analyzed without adjustments (base model), with adjustment for cohort, maternal smoking and maternal hypertensive disorders (model 1) and with adjustment for cohort, maternal smoking, maternal hypertensive disorders, pre-pregnancy BMI, SES, parity and maternal age at delivery (model 2). Statistical analyses were conducted using $\mathrm{R}$ software package version 3.5.0 [18].

\section{Results}

\section{Characteristics of the sample population}

Distribution of offspring sex, maternal age and parity were similar between the cohorts (Table 1 ). Other maternal and fetal characteristics changed within the 20 years between the cohort initiations. Number of mothers with low $\mathrm{mHb}$ at any point of pregnancy was lower in NFBC1966 than in NFBC1986 whereas number of housewives was considerably higher in NFBC1966. Furthermore, number of mothers with either gestational or chronic hypertension was higher in NFBC1966.

\section{Multinomial logistic regression for perinatal outcomes}

As shown in Table 2, low mHb at late pregnancy associated with increased risk of prematurity (fully adjusted OR (aOR) 1.60, 95\% CI $[1.26,2.02], \mathrm{p}<0.0005)$. Associations between $\mathrm{mHb}$ and SGA and LGA are indicated in Table 3. Low $\mathrm{mHb}$ at early pregnancy associated with decreased risk of SGA (aOR 0.73, 95\% CI [0.58, 0.93], $\mathrm{p}=0.010)$ and high $\mathrm{mHb}$ at late pregnancy with increased risk of SGA (aOR 1.29, 95\% CI [1.07, 1.56], $\mathrm{p}=0.009$, Table 2). Low mHb at early pregnancy associated with increased risk of LGA; however, the association was not statistically significant after adjustment for maternal cofactors. High $\mathrm{mHb}$ at early pregnancy associated with decreased risk of LGA in fully adjusted model but not in other models. Results are shown separately for each cohort in Supplementary Table 1 . To characterize spontaneous preterm delivery and normotensive SGA, we conducted additional MLR analyses for pregnancies without maternal hypertensive disorders

Table 2

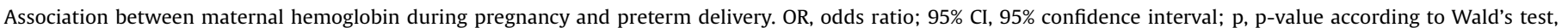
significant when $\mathrm{p}<0.0125$.

\begin{tabular}{|c|c|c|c|c|c|c|c|c|c|c|}
\hline \multirow[b]{2}{*}{ Outcome } & \multirow[b]{2}{*}{$\mathrm{mHb}$} & \multicolumn{2}{|c|}{ Base model } & \multirow[b]{2}{*}{$\mathrm{p}$} & \multicolumn{2}{|c|}{ Model $1^{\mathrm{a}}$} & \multirow[b]{2}{*}{$\mathrm{p}$} & \multicolumn{2}{|c|}{ Model $2^{b}$} & \multirow[b]{2}{*}{$\mathrm{p}$} \\
\hline & & OR & $95 \% \mathrm{CI}$ & & OR & $95 \% \mathrm{CI}$ & & OR & $95 \% \mathrm{CI}$ & \\
\hline \multirow{4}{*}{$\begin{array}{l}\text { Preterm } \\
\text { delivery }\end{array}$} & Low early & 1.17 & {$[0.94,1.44]$} & 0.157 & 1.23 & {$[0.97,1.56]$} & 0.081 & 1.24 & {$[0.97,1.59]$} & 0.079 \\
\hline & High early & 1.12 & {$[0.90,1.39]$} & 0.323 & 1.10 & {$[0.86,1.41]$} & 0.437 & 1.18 & {$[0.91,1.52]$} & 0.209 \\
\hline & Low late & 1.58 & {$[1.29,1.93]$} & $<\mathbf{0 . 0 0 0 5}$ & 1.64 & {$[1.31,2.05]$} & $<0.0005$ & 1.60 & {$[1.26,2.02]$} & $<\mathbf{0 . 0 0 0 5}$ \\
\hline & High late & 0.87 & {$[0.67,1.12]$} & 0.275 & 0.81 & {$[0.61,1.09]$} & 0.166 & 0.83 & {$[0.62,1.13]$} & 0.236 \\
\hline
\end{tabular}

\footnotetext{
a Model 1 adjusted for cohort, maternal smoking and maternal hypertensive disorders.

b Model 2 adjusted for cohort, maternal smoking, maternal hypertensive disorders, pre-pregnancy BMI, maternal SES, parity and maternal age.
} 
Table 3

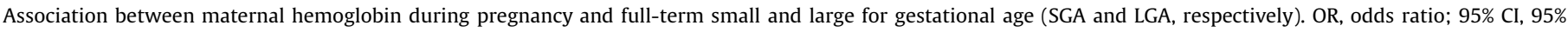
confidence interval; p, p-value according to Wald's test, significant when $\mathrm{p}<0.0125$.

\begin{tabular}{|c|c|c|c|c|c|c|c|c|c|c|}
\hline \multirow[b]{2}{*}{ Outcome } & \multirow[b]{2}{*}{$\mathrm{mHb}$} & \multicolumn{2}{|c|}{ Base model } & \multirow[b]{2}{*}{$\mathrm{p}$} & \multicolumn{2}{|c|}{ Model $1^{\mathrm{a}}$} & \multirow[b]{2}{*}{$\mathrm{p}$} & \multicolumn{2}{|c|}{ Model $2^{\mathrm{b}}$} & \multirow[b]{2}{*}{$\mathrm{p}$} \\
\hline & & OR & $95 \% \mathrm{CI}$ & & OR & $95 \% \mathrm{CI}$ & & OR & $95 \% \mathrm{CI}$ & \\
\hline \multirow[t]{4}{*}{ SGA } & Low early & 0.74 & {$[0.61,0.90]$} & 0.003 & 0.75 & {$[0.59,0.94]$} & 0.011 & 0.73 & {$[0.58,0.93]$} & 0.010 \\
\hline & High early & 1.10 & {$[0.93,1.30]$} & 0.280 & 1.07 & {$[0.87,1.31]$} & 0.518 & 1.15 & {$[0.93,1.42]$} & 0.199 \\
\hline & Low late & 0.88 & {$[0.74,1.06]$} & 0.173 & 0.82 & {$[0.67,1.02]$} & 0.073 & 0.84 & {$[0.67,1.05]$} & 0.129 \\
\hline & High late & 1.29 & {$[1.10,1.50]$} & 0.001 & 1.30 & {$[1.09,1.56]$} & 0.004 & 1.29 & {$[1.07,1.56]$} & 0.009 \\
\hline \multirow[t]{4}{*}{ LGA } & Low early & 1.23 & {$[1.05,1.44]$} & 0.010 & 1.25 & {$[1.05,1.50]$} & 0.013 & 1.23 & {$[1.02,1.49]$} & 0.030 \\
\hline & High early & 0.95 & {$[0.80,1.14]$} & 0.598 & 0.83 & {$[0.68,1.03]$} & 0.086 & 0.72 & {$[0.57,0.91]$} & 0.005 \\
\hline & Low late & 1.22 & {$[1.04,1.43]$} & 0.014 & 1.25 & {$[1.04,1.50]$} & 0.015 & 1.22 & {$[1.01,1.48]$} & 0.039 \\
\hline & High late & 0.96 & {$[0.81,1.13]$} & 0.605 & 0.93 & {$[0.76,1.12]$} & 0.436 & 0.99 & {$[0.81,1.21]$} & 0.915 \\
\hline
\end{tabular}

${ }^{\text {a }}$ Model 1 adjusted for cohort, maternal smoking and maternal hypertensive disorders.

b Model 2 adjusted for cohort, maternal smoking, maternal hypertensive disorders, pre-pregnancy BMI, maternal SES, parity and maternal age.

as these have been shown to associate with preterm delivery as well as SGA (reviewed in [19]). As shown in Supplementary Table 2, similar to main analyses, low $\mathrm{mHb}$ at late pregnancy was associated with increased risk of spontaneous preterm delivery (aOR 1.65, 95\% CI [1.27, 2.15], p < 0.0005). High $\mathrm{mHb}$ at late pregnancy was associated with increased risk of normotensive full-term SGA in model 1 (OR 1.34, 95\% CI [1.08, 1.66], $\mathrm{p}=0.009$ ) and borderline significant in other models.

\section{Sensitivity analyses}

As we do not have the exact gestational age at the last antenatal visit for all mothers, we were not able to estimate the effect of exact $\mathrm{mHb}$ measurement timing on the perinatal outcome. This is critical specifically with the case of prematurity, because during normal pregnancy, $\mathrm{mHb}$ decreases until 20 weeks of gestation and then begins to increase around 30 weeks of gestation [20]. To confirm that our results are not due to normal $\mathrm{mHb}$ change during pregnancy, we conducted sensitivity analyses such as i) MLR of individuals with available information about gestational age at last antenatal visit ( $n=7,281$ ), ii) MLR of pregnancies with birth at 32 weeks of gestation or more and iii) MLR of gestational age-adjusted late pregnancy $\mathrm{mHb}$. These analyses showed similar results with the main analyses (Supplementary Tables 3-5). Association between prematurity and low $\mathrm{mHb}$ at late pregnancy was not statistically significant when gestational age at last antenatal visit was added to the models (Supplementary Table 3). This is most likely due to lack of power because only 45 preterm deliveries had the information about gestational age at last antenatal visit and were included in the gestational age at last antenatal visit-adjusted model.

\section{Comment}

The current study indicates that low $\mathrm{mHb}$ at late pregnancy is associated with increased risk of preterm delivery and high $\mathrm{mHb}$ with increased risk of full-term SGA. These results support the previously published concept of U-shaped association between $\mathrm{mHb}$ concentration and the risk of adverse perinatal outcomes [7,21]. However, there are discrepancies between previous publications with respect of this association. One problem related to the generalization of the results from $\mathrm{mHb}$ studies concerns the differences between study populations [22]. In low-income countries, the $\mathrm{mHb}$ level often relates to the poor nutritional status of the mother contributing to adverse perinatal outcomes. In addition, the prevalence of infectious diseases such as HIV and malaria is higher in the developing countries, which adds further burden to maternal health [5]. This socioeconomic and nutritional disparity may lead to severe residual confounding in the analyses. Current study population is located in Finland where standardized antenatal care system and relatively high living standards induce smaller differences in environmental factors affecting the development of the fetus and yet still, abnormal mHb was associated with prematurity and SGA.

Another issue varying between studies relates to the timing of the mHb measurement during pregnancy. Level of $\mathrm{mHb}$ changes naturally throughout the pregnancy and it may be critical for the results whether the measurement occurs at early or late pregnancy [20]. In the current study, we wanted to evaluate $\mathrm{mHb}$ at early and late pregnancy to find out how they contribute to overall risk of adverse perinatal outcomes. Our results indicate that abnormal $\mathrm{mHb}$ measured at late pregnancy associates with increased risk of prematurity and SGA. Fetus grows rapidly during the third trimester when iron and other micronutrient demands are specifically high [23]. Abnormal $\mathrm{mHb}$ may reflect insufficient availability and/or delivery of nutrients to the fetus and specifically at the end of pregnancy alter the rapid growth phase. It is safe to assume that due to systematic antenatal care in Finland, if low $\mathrm{mHb}$ was measured at the beginning of the pregnancy, it had been attempted to increase by administration of iron supplements or other medical actions. Interestingly, low $\mathrm{mHb}$ at the beginning of pregnancy was in fact associated with decreased risk of SGA in current study. In high-income countries, approximately $60 \%$ of the pregnant women with low hemoglobin are estimated to be amenable to iron supplementation [9]. Thus, the mothers with low $\mathrm{mHb}$ at late pregnancy may be the ones that do not response to iron supplementation and are at increased risk of preterm delivery in the current study. We know that iron supplementation was recommended already in 1960s for pregnant women to prevent gestational anemia [8,24]; however, we do not have information about its administration or iron status of the mother in the current study to research this issue in more detail.

Further variation within mHb studies is added by varying cutoffs for categorical variables of low and high $\mathrm{mHb}$. WHO defines anemia during pregnancy as mHb less than $110 \mathrm{~g} / \mathrm{l}$, which is widely used cutoff for low mHb [9]. However, discussion about an appropriate cutoff during pregnancy is ongoing as some studies found association only with mHb levels below $100 \mathrm{~g} / \mathrm{l}[25,26]$. Furthermore, similar to previous studies located in Finland and Sweden [22,27], the prevalence of anemia with the $110 \mathrm{~g} / \mathrm{l}$ cutoff was low in the current study, only approximately $4 \%$. The earlier Finnish study with more stringent cutoff of $100 \mathrm{~g} / \mathrm{l}$ did not found association between $\mathrm{mHb}$ and preterm delivery and SGA, which may result from decreased statistical power due to low number of mothers with "true anemia" [22]. To overcome this issue, we decided to define lowest $10 \%$ of the $\mathrm{mHb} z$ values as low $\mathrm{mHb}$ and highest $10 \%$ of the $\mathrm{mHb} z$ values as high $\mathrm{mHb}$. These correspond to cutoff values for low mHb of approximately $110 \mathrm{~g} / \mathrm{l}$ in NFBC1966 and $117 \mathrm{~g} / \mathrm{l}$ in NFCB1986. For high $\mathrm{mHb}$, cutoff values were $135 \mathrm{~g} / \mathrm{l}$ and $140 \mathrm{~g} / \mathrm{l}$ in NFBC1966 and NFBC1986, respectively. Z values were calculated for each cohort and pregnancy time point separately, thus the extremes of $\mathrm{mHb}$ are relative for the reference, not for the 
absolute mHb concentration that may differ throughout the pregnancy.

The mechanism behind the association is unclear. Iron deficiency may contribute to the restricted fetal growth and prematurity through such mechanisms as increased maternal and fetal stress, increased serum norepinephrine production and oxidative damage to erythrocytes and fetoplacental unit (reviewed in [28]). Mothers with low mHb show increased placental vascularization suggesting earlier placental maturity, which may contribute to increased risk of preterm delivery [29,30]. Low mHb may cause chronic hypoxia which in turn may induce a stressresponse with increased placental corticotropin-releasing hormone $(\mathrm{CRH})$ production. During normal pregnancy, a rapid increase in bioavailable $\mathrm{CRH}$ induces labor and if this occurs too early, it may induce preterm delivery (28). High $\mathrm{mHb}$, specifically during late pregnancy may indicate incomplete hemodilution and may lead to fetal hypoxia due to increased blood viscosity and impairment of maternal-fetal exchange and contribute to the risk of SGA [1 ]. Further studies located in high-income countries as well as deeper mechanistic understanding are needed with respect of association between $\mathrm{mHb}$ and fetal growth.

Strengths and weaknesses of the study

Large sample size in a homogenous population is major strength of the current study. Universal antenatal care available in Finland and high standard of living offer less variation in the environmental factors affecting the intrauterine development of the child. First limitation is that we do not have reliable information about exact gestational age at $\mathrm{mHb}$ measurements from all study subjects in the current study. The measurement timing is specifically important when prematurity is assessed because $\mathrm{mHb}$ begins to naturally increase after 30 weeks of gestation. To overcome this, we conducted series of sensitivity analyses that showed similar results as our main models indicating that the association between low $\mathrm{mHb}$ at late pregnancy and prematurity is not mediated by the timing of $\mathrm{mHb}$ measurement. Other limitations relate to the lack of clinical measures of fetal hypoxia and iron metabolism. Our data suggests that there may be distinct outcomes depending on the mother's responsiveness on iron supplementation. The current study shows that both low and high $\mathrm{mHb}$ may indicate increased risk for distress in the developing child. Mechanistic studies are warranted to reveal the possible causal influence of abnormal $\mathrm{mHb}$ on perinatal outcomes.

\section{Conflict of interest}

The authors report no conflict of interest.

\section{Source of funding}

This work was supported by the European Union's Horizon 2020 research and innovation program under grant agreement No. 633595 (DynaHEALTH), and grant agreement No. 733206 (LifeCycle); the academy of Finland EGEA-project (285547) and the Biocenter Oulu.

\section{Appendix A. Supplementary data}

Supplementary data associated with this article can be found, in the online version, at https://doi.org/10.1016/j.ejogrb.2019.04.045.

\section{References}

[1] Cordina M, Bhatti S, Fernandez M, Syngelaki A, Nicolaides KH, Kametas NA. Association between maternal haemoglobin at 27-29 weeks gestation and intrauterine growth restriction. Pregnancy Hypertens 2015;5(October (4)):339-45.
[2] Gonzales GF, Steenland K, Tapia V. Maternal hemoglobin level and fetal outcome at low and high altitudes. Am J Physiol Regul Integr Comp Physiol 2009;297(November (5)):R1477-85.

[3] Jwa SC, Fujiwara T, Yamanobe Y, Kozuka K, Sago H. Changes in maternal hemoglobin during pregnancy and birth outcomes. BMC Pregnancy Childbirth 2015;2(April (15))80 015-0516-1.

[4] Tandu-Umba B, Mbangama AM. Association of maternal anemia with other risk factors in occurrence of Great obstetrical syndromes at university clinics, Kinshasa, DR Congo. BMC Pregnancy Childbirth 2015;21(August (15))183 015-0623-z.

[5] Rahman MM, Abe SK, Rahman MS, Kanda M, Narita S, Bilano V, et al. Maternal anemia and risk of adverse birth and health outcomes in low- and middleincome countries: systematic review and meta-analysis. Am J Clin Nutr 2016;103(February (2)):495-504.

[6] Levy A, Fraser D, Katz M, Mazor M, Sheiner E. Maternal anemia during pregnancy is an independent risk factor for low birthweight and preterm delivery. Eur J Obstet Gynecol Reprod Biol 2005;122(October (2)):182-6.

[7] Scanlon KS, Yip R, Schieve LA, Cogswell ME. High and low hemoglobin levels during pregnancy: differential risks for preterm birth and small for gestational age. Obstet Gynecol 2000;96(November (5 Pt 1)):741-8.

[8] Fararouei M, Robertson C, Whittaker J, Sovio U, Ruokonen A, Pouta A, et al Maternal $\mathrm{Hb}$ during pregnancy and offspring's educational achievement: a prospective cohort study over 30 years. Br J Nutr 2010;104(November (9)):1363-8.

[9] WHO. The global prevalence of anaemia in 2011. Geneva: World Health Organization; 2015.

[10] Stevens GA, Finucane MM, De-Regil LM, Paciorek CJ, Flaxman SR, Branca F, et al Global, regional, and national trends in haemoglobin concentration and prevalence of total and severe anaemia in children and pregnant and nonpregnant women for 1995-2011: a systematic analysis of populationrepresentative data. Lancet Glob Health 2013;1(July (1):e16-25.

[11] Salihu HM, Wilson RE. Epidemiology of prenatal smoking and perinatal outcomes. Early Hum Dev 2007:83(November (11)):713-20.

[12] Milman N, Pedersen AN. Blood haemoglobin concentrations are higher in smokers and heavy alcohol consumers than in non-smokers and abstainers: should we adjust the reference range? Ann Hematol 2009;88(July (7)):687-94.

[13] Hendler I, Goldenberg RL, Mercer BM, Iams JD, Meis PJ, Moawad AH, et al. The Preterm Prediction Study: association between maternal body mass index and spontaneous and indicated preterm birth. Am J Obstet Gynecol 2005;192 (March (3)):882-6.

[14] Ruiz M, Goldblatt P, Morrison J, Kukla L, Svancara J, Riitta-Jarvelin M, et al Mother's education and the risk of preterm and small for gestational age birth: a DRIVERS meta-analysis of 12 European cohorts. J Epidemiol Community Health 2015;69(September (9)):826-33.

[15] Kozuki N, Lee AC, Silveira MF, Sania A, Vogel JP, Adair L, et al. The associations of parity and maternal age with small-for-gestational-age, preterm, and neonatal and infant mortality: a meta-analysis. BMC Public Health 2013;13(Suppl. 3)S2 2458-13-S3-S2. Epub 2013 Sep 17.

[16] Pihkala J, Hakala T, Voutilainen P, Raivio K. Characteristic of recent fetal growth curves in Finland. Duodecim 1989;105(18):1540-6.

[17] Mannisto T, Karumanchi SA, Pouta A, Vaarasmaki M, Mendola P, Miettola S, et al. Preeclampsia, gestational hypertension and subsequent hypothyroidism. Pregnancy Hypertens 2013;3(January (1)):21-7.

[18] Team RC. R: a language and environment for statistical computing. 2018.

[19] Vest AR, Cho LS. Hypertension in pregnancy. Curr Atheroscler Rep 2014;16 (March (3))395 013-0395-8.

[20] Steer PJ. Maternal hemoglobin concentration and birth weight. Am J Clin Nutr 2000;71(May (Suppl. 5)):1285S-7S.

[21] Dewey KG, Oaks BM. U-shaped curve for risk associated with maternal hemoglobin, iron status, or iron supplementation. Am J Clin Nutr 2017 (October):25.

[22] Hamalainen H, Hakkarainen K, Heinonen S. Anaemia in the first but not in the second or third trimester is a risk factor for low birth weight. Clin Nutr 2003;22 (June (3)):271-5.

[23] Kumar KJ, Asha N, Murthy DS, Sujatha M, Manjunath V. Maternal anemia in various trimesters and its effect on newborn weight and maturity: an observational study. Int J Prev Med 2013;4(February (2)):193-9.

[24] Nevanlinna HR. Therapy of anemia. Duodecim 1965;81:141-5.

[25] Kozuki N, Lee AC, Katz J, Child Health Epidemiology Reference Group. Moderate to severe, but not mild, maternal anemia is associated with increased risk of small-for-gestational-age outcomes. J Nutr 2012;142 (February (2)):358-62.

[26] Gonzales GF, Tapia V, Gasco M, Carrillo CE. Maternal hemoglobin concentration and adverse pregnancy outcomes at low and moderate altitudes in Peru. Matern Fetal Neonatal Med 2012;25(July (7)):1105-10.

[27] Stephansson O, Dickman PW, Johansson A, Cnattingius S. Maternal hemoglobin concentration during pregnancy and risk of stillbirth. JAMA 2000;284 (November (20)):2611-7.

[28] Allen LH. Biological mechanisms that might underlie iron's effects on fetal growth and preterm birth. J Nutr 2001;131(February (2S-2)):581S-9S.

[29] Lelic M, Bogdanovic G, Ramic S, Brkicevic E. Influence of maternal anemia during pregnancy on placenta and newborns. Med Arch 2014;68(June (3)):184-7.

[30] Stangret A, Wnuk A, Szewczyk G, Pyzlak M, Szukiewicz D. Maternal hemoglobin concentration and hematocrit values may affect fetus development by influencing placental angiogenesis. J Matern Fetal Neonatal Med 2017;30(January (2)):199-204. 